Pacific Journal of Mathematics

AN ANALOG OF THE MINIMAX THEOREM FOR VECTOR 


\title{
AN ANALOG OF THE MINIMAX THEOREM FOR VECTOR PAYOFFS
}

\author{
DAVID BLACKWELL
}

1. Introduction. The von Neumann minimax theorem [2] for finite games asserts that for every $r \times s$ matrix $M=\|m(i, j)\|$ with real elements there exist a number $v$ and vectors

$$
p=\left(p_{1}, \cdots, p_{r}\right), q=\left(q_{1}, \cdots, q_{s}\right), \quad p_{i}, q_{j} \geqq 0, \quad \sum p_{i}=\sum q_{j}=1
$$

such that

$$
\sum_{i} p_{i} m(i, j) \geqq v \geqq \sum_{j} q_{j} m(i, j)
$$

for all $i, j$. Thus in the (two-person, zero-sum) game with matrix $M$, player I has a strategy insuring an expected gain of at least $v$, and player II has a strategy insuring an expected loss of at most $v$. An alternative statement, which follows from the von Neumann theorem and an appropriate law of large numbers is that, for any $\varepsilon>0$, I can, in a long series of plays of the game with matrix $M$, guarantee, with probability approaching 1 as the number of plays becomes infinite, that his average actual gain per play exceeds $v-\varepsilon$ and that II can similarly restrict his average actual loss to $v+\varepsilon$. These facts are assertions about the extent to which each player can control the center of gravity of the actual payoffs in a long series of plays. In this paper we investigate the extent to which this center of gravity can be controlled by the players for the case of matrices $M$ whose elements $m(i, j)$ are points of $N$-space. Roughly, we seek to answer the following question. Given a matrix $M$ and a set $S$ in $N$-space, can I guarantee that the center of gravity of the payoffs in a long series of plays is in or arbitrarily near $S$, with probability approaching 1 as the number of plays becomes infinite? The question is formulated more precisely below, and a complete solution is given in two cases: the case $N=1$ and the case of convex $S$.

Let

$$
M=\|m(i, j)\|,
$$$$
1 \leqq i \leqq r, 1 \leqq j \leqq s
$$

be an $r \times s$ matrix, each element of which is a probability distribution over a closed bounded convex set $X$ in Euclidean $N$-space. By a strategy for Player I is meant a sequence $f=\left\{f_{n}\right\}, n=0,1,2, \ldots$ of functions, where $f_{n}$ is defined on the set of $n$-tuples $\left(x_{1}, \cdots, x_{n}\right), x_{i} \in X$

Received September 14, 1954. This paper was written under contract Nonr 1197(00) with the Office of Naval Research. 
and has values in the set $P$ of vectors $p=\left(p_{1}, \cdots, p_{r}\right)$ with $p_{i} \geq 0, \sum_{1}^{r} p_{i}=$ $1 ; f_{0}$ is simply a point in $P$. A strategy $g=\left\{g_{n}\right\}$ for Player II is defined similarly, except that the values of $g_{n}$ are in the set $Q$ of vectors $q=$ $\left(q_{1}, \cdots, q_{s}\right)$ with $q_{j} \geqq 0, \sum_{1}^{s} q_{j}=1$. The interpretation is that I, II select $i, j$ according to the distributions $f_{0}, g_{0}$ respectively, and a point $x_{1} \in X$ is selected according to the distribution $m(i, j)$. The players are told $x_{1}$, after which they again select $i, j$, this time according to the distributions $f_{1}\left(x_{1}\right), g_{1}\left(x_{1}\right)$, a point $x_{2}$ is chosen according to the $m(i, j)$ corresponding to their second choices, they are told $x_{2}$ and select a third $i, j$ according to $f_{2}\left(x_{1}, x_{2}\right), g_{2}\left(x_{1}, x_{2}\right)$, etc. Thus each pair $(f, g)$ of strategies, together with $M$, determines a sequence of (vector-valued) random variables $x_{1}, x_{2}, \cdots$.

Let $S$ be any set in $N$-space. We shall say that $S$ is approachable with $f^{*}$ in $M$, if for every $\varepsilon>0$ there is an $N_{0}$ such that, for every $g$,

$$
\text { Prob }\left\{\delta_{n} \geqq \varepsilon \text { for some } n \geqq N_{0}\right\}<\varepsilon,
$$

where $\delta_{n}$ denotes the distance of the point $\sum_{1}^{n} x_{i} / n$ from $S$ and $x_{1}, x_{2}$, $\cdots$ are the variables determined by $f^{*}, g$. We shall say that $S$ is $e x-$ cludable with $g^{*}$ in $M$, if there exists $d>0$ such that for every $\varepsilon>0$ there is an $N_{0}$ such that, for every $f$,

$$
\text { Prob }\left\{\delta_{n} \geqq d \text { for all } n \geqq N_{0}\right\}>1-\varepsilon \text {, }
$$

where $x_{1}, x_{2}, \cdots$ are the variables determined by $f, g^{*}$. We shall say that $S$ is approachable (excludable) in $M$, if there exists $f^{*}\left(g^{*}\right)$ such that $S$ is approachable with $f^{*}$ (excludable with $g^{*}$ ). Approachability and excludability are clearly the same for $S$ and its closure, so that we may suppose $S$ closed.

In terms of these concepts, von Neumann's theorem has the following analog.

For $N=1$, associated with every $M$ are $a$ number $v$ and vectors $p \in P$, $q \in Q$ such that the set $S=\{x \geqq t\}$ is approachable for $t \leqq v$ with $f: f_{n} \equiv p$ and excludable for $t>v$ with $g: g_{n} \equiv q$.

A slightly more complete result for $N=1$, characterizing all approachable and excludable sets $S$ for a given $M$, is given in $\S 4$ below.

Obviously any superset of an approachable set is approachable, any subset of an excludable set is excludable, and no set is both approachable and excludable. Another obvious fact which will be useful is that if a closed set $S$ is approachable in the $s \times r$ matrix $M^{\prime}$, the transpose of $M$, then any closed set $T$ not intersecting $S$ is excludable in $M$ with any strategy with which $S$ is approachable in $M^{\prime}$. Thus any sufficient condition for approachability yields immediately a sufficient condition for excludability. A sufficient condition for approachability is given in $\S 2$. It turns out that every convex $S$ satisfies either this condition for 
approachability or the corresponding condition for excludability, enabling us to give in $\S 3$ a complete solution for convex $S$. For non-convex $S$, the problem is not solved except for $N=1$. An example of a set which is neither approachable nor excludable in a given $M$ is given in $\S 5$, the concepts of weak approachability and excludability are introduced, and it is conjectured that every set is either weakly approachable or weakly excludable.

2. A sufficient condition for approachability. If $x, y$ are distinct points in $N$-space, $H$ is the hyperplane through $y$ perpendicular to the line segment $x y$, and $z$ is any point on $H$ or on the opposite side of $H$ from $x$, then all points interior to the line segment $x z$ and sufficiently near $x$ are closer to $y$ than is $x$. This fact is the basis for our sufficient condition for approachability.

For any matrix $M$, denote by $\bar{M}$ the matrix whose elements $\bar{m}(i, j)$ are the mean values of the distributions $m(i, j)$. For any $p \in P$ denote by $R(p)$ the convex hull of the $s$ points $\sum_{i} p_{i} \bar{m}(i, j)$. The sufficient condition for approachability is given in the following theorem.

THeOREm 1. Let $S$ be any closed set. If for every $x \notin S$ there is a $p(=p(x)) \in P$ such that the hyperplane through $y$, the closest point in $S$ to $x$, perpendicular to the line segment $x y$ separates $x$ from $R(p)$, then $S$ is approachable with the strategy $f: f_{n}$, where

$$
f_{n}=\left\{\begin{array}{l}
p\left(\bar{x}_{n}\right) \text { if } n>0 \text { and } \bar{x}_{n}=\left(\frac{1}{n} \sum_{1}^{n} x_{i}\right) \notin S \\
\text { arbitrary if } n=0 \text { or } \bar{x}_{n} \in S .
\end{array}\right.
$$

Proof. Suppose the hypotheses satisfied, let I use the specified strategy, let II use any strategy, and let $x_{1}, x_{2}, \ldots$ be the resulting sequence of chance variables. For

$$
\bar{x}_{n}=\left(\frac{1}{n} \sum_{1}^{n} x_{i}\right) \notin S,
$$

let $y_{n}$ be the point of $S$ closest to $\bar{x}_{n}$, and write $u_{n}=y_{n}-\bar{x}_{n}$. Then, for $\bar{x}_{n} \notin S$,

$$
E\left(\left(u_{n}, x_{n+1}\right) \mid x_{1}, \cdots, x_{n}\right) \geq\left(u_{n}, y_{n}\right),
$$

where $E(x \mid y)$ denotes the conditional expectation of $x$ given $y$ and $(u, v)$ denotes the inner product of the vectors $u$ and $v$.

Let $\delta_{n}$ denote the squared distance from $\bar{x}_{n}$ to $S$. If $\delta_{n}>0$, then

$$
\delta_{n+1} \leqq\left|\bar{x}_{n+1}-y_{n}\right|^{2}=\left|\bar{x}_{n}-y_{n}\right|^{2}+2\left(\bar{x}_{n}-y_{n}, \bar{x}_{n+1}-\bar{x}_{n}\right)+\left|\bar{x}_{n+1}-\bar{x}_{n}\right|^{2} \text {. }
$$


Since $\bar{x}_{n+1}-\bar{x}_{n}=\left(x_{n+1}-\bar{x}_{n}\right) /(n+1)$, we have

$$
\left(\bar{x}_{n}-y_{n}, \bar{x}_{n+1}-\bar{x}_{n}\right)=\frac{\left(\bar{x}_{n}-y_{n}, x_{n+1}-y_{n}\right)}{n+1}+\frac{\left(\bar{x}_{n}-y_{n}, y_{n}-\bar{x}_{n}\right)}{n+1}
$$

and

$$
\left|\bar{x}_{n+1}-\bar{x}_{n}\right|^{2} \leqq c /(n+1)^{2},
$$

where $c$ depends only on the size of the bounded set $X$. From (2), using (1), (3), and (4), we obtain, replacing $n$ by $n-1$,

$$
E\left(\delta_{n} \mid \delta_{1}, \cdots, \delta_{n-1}\right) \leqq\left(1-\frac{2}{n}\right) \delta_{n-1}+\frac{c}{n^{2}} \quad \text { if } \delta_{n-1}>0 .
$$

Moreover

$$
0 \leqq \delta_{n} \leqq a
$$

and

$$
\left|\delta_{n}-\delta_{n-1}\right| \leqq \frac{b}{n}
$$

Thus it remains only to establish the following.

LEMma. A sequence of chance variables $\delta_{1}, \delta_{2}, \cdots$ satisfying (5), (6), and (7) converges to zero with probability 1 at a rate depending only on $a, b, c$, that is, for every $\varepsilon>0$ there is an $N_{0}$ depending only on $\varepsilon$, $a, b, c$ such that for auy $\left\{\delta_{n}\right\}$ satisfying (5), (6), and (7), we have

$$
\text { Prob }\left\{\delta_{n} \geq \varepsilon \text { for some } n \geq N_{0}\right\}<\varepsilon .
$$

Proof of Lemma. Let $n_{0}$ be any integer. There exists $n_{1}>n_{0}$, depending only on $n_{0}, \varepsilon, a, c$ such that

$$
\text { Prob }\left\{\delta_{n} \geqq \varepsilon / 2 \text { for } n_{0} \leqq n \leqq n_{1}\right\}<\varepsilon / 2 .
$$

To see this, define, for $n \geqq n_{0}, \alpha_{n}=\delta_{n}$ if $\delta_{i}>0$ for $n_{0} \leqq i \leqq n$, and $\alpha_{n}=0$ otherwise. Then $\alpha_{n}<\varepsilon / 2$ implies $\delta_{i}<\varepsilon / 2$ for some $i$ with $n_{0} \leqq i \leqq n$. Also $\alpha_{n_{0}} \leqq a$ and, for $n>n_{0}$,

$$
E\left(\alpha_{n} \mid \alpha_{n_{0}}, \cdots, \alpha_{n-1}\right) \leqq\left(1-\frac{2}{n}\right) \alpha_{n-1}+\frac{c}{n^{2}},
$$

so that

$$
E\left(\alpha_{n}\right) \leqq\left(1-\frac{2}{n}\right) E\left(\alpha_{n-1}\right)+\frac{c}{n^{2}}
$$


Thus $E\left(\alpha_{n}\right) \rightarrow 0$ at a rate depending only on $n_{0}, a, c$, and there is an $n_{1}$ depending only on $n_{0}, \varepsilon, a, c$ for which $E\left(\alpha_{n_{1}}\right)$ is so small that

$$
\text { Prob }\left\{\alpha_{n_{1}}<\varepsilon / 2\right\}>1-(\varepsilon / 2) \text {. }
$$

For every $n, k$ with $n \leqq k$ we define variables $z_{n k}$ as follows. Unless $\delta_{n-1}<\varepsilon / 2$ and $\delta_{n} \geqq \varepsilon / 2, z_{n k}=0$ for all $k$. If $\delta_{n-1}<\varepsilon / 2$ and $\delta_{i} \geqq \varepsilon / 2$ for $n \leqq i \leqq k$, then $z_{n k}=\delta_{k}$. If $\delta_{n-1}<\varepsilon / 2, \delta_{i} \geqq \varepsilon / 2$ for $n \leqq i<k_{0}$ and $\delta_{k_{0}}<\varepsilon / 2$, then $z_{n k}+z_{n k_{0}}=\delta_{k_{0}}$ for $k \geqq k_{0}$. If $\delta_{n} \geqq \varepsilon$ for some $n \geqq n_{1}$, either $\delta_{n} \geqq \varepsilon / 2$ for all $n$ such that $n_{0} \leqq n \leqq n_{1}$ or $z_{n k} \geq \varepsilon$ for some $n \geqq n_{0}$. The former event has already been shown to have probability less than $\varepsilon / 2$; it remains to show that the probability of the latter event can be made less than $\varepsilon / 2$ by choosing $n_{0}$ sufficiently large.

Fix $n \geqq n_{0}$ and write $\beta_{k}=z_{n k}-z_{n k-1}, k>n, \beta_{n}=0$. Then, if $z_{n k-1} \geqq \varepsilon / 2$

$$
E\left(\beta_{k} \mid z_{n n}, \beta_{n}, \cdots, \beta_{k-1}\right) \leqq-\frac{2}{k} z_{n k-1}+\frac{c}{k^{2}} \leqq-\frac{\varepsilon}{2 k}
$$

for sufficiently large $n_{0}$ depending on $c$ and $\varepsilon$, and $\left|\beta_{k}\right| \leqq b / k$. If $z_{n k-1}<$ $\varepsilon / 2, \beta_{k}=0$ so that, in any case

$$
E\left(\beta_{k} \mid \beta_{n}, \cdots, \beta_{k-1}\right) \leqq-\frac{\varepsilon}{2 b} \max \left(\mid \beta_{k} \| \beta_{n}, \cdots, \beta_{k-1}\right)
$$

We now apply the following form of the strong law of large numbers, recently proved by the writer [1].

THEOREM 2. If $z_{1}, z_{2}, \cdots$ is a sequence of random variables such that $\left|z_{k}\right| \leqq 1$ and

$$
E\left(z_{k} \mid z_{1}, \cdots, z_{k-1}\right) \leqq-u \max \left(\mid z_{k} \| z_{1}, \cdots, z_{k-1}\right), \quad u>0,
$$

then for all $t$,

Prob $\left\{z_{1}+\cdots+z_{k} \geqq t\right.$ for some $\left.k\right\} \leqq\left(\frac{1-u}{1+u}\right)^{t}$.

The variables $z_{k}=(n / b) \beta_{k-n+1}$ satisfy the hypotheses of Theorem 2, with $u=(\varepsilon / 2 b)$, so that

$$
\text { Prob }\left\{z_{n k}-z_{n n}>t \text { for some } k \geqq n\right\} \leqq r^{t n}, \quad r=\left(\frac{1-u}{1+u}\right)^{1 / b},
$$

For large $n_{0}, z_{n n}<3 \varepsilon / 4$, so that $z_{n k} \geqq \varepsilon$ for some $k$ implies $z_{n k}-z_{n n}>\varepsilon / 4$. Thus

$$
\text { Prob }\left\{z_{n k} \geqq \varepsilon \text { for some } k \geqq n\right\} \leqq s^{n},
$$

where $s=r^{\varepsilon / 4}$, so that 
Prob $\left\{z_{n k \geqq \varepsilon}\right.$ for some $\left.n \geqq n_{0}, k \geqq n\right\} \leqq \sum_{n_{0}}^{\infty} s^{n}$,

which will be less than $\varepsilon / 2$ for $n_{0}$ sufficiently large. This completes the proof.

\section{The case of convex $S$.}

THEOREM 3. Let $T(q)$ denote the convex hull of the $r$ points $\sum_{1}^{s} q_{j} \bar{m}(i, j) . \quad A$ closed convex set $S$ is approachable if and only if it intersects every set $T(q)$. If it fails to intersect $T\left(q_{0}\right)$, it is excludable with $g: g_{n} \equiv q_{0}$.

Proof. Suppose $S$ intersects every $T(q)$, let $x_{0} \notin S$, let $y$ be the point of $S$ closest to $x_{0}$, and consider the game with matrix $A=\|a(i, j)\|$, where $a(i, j)=\left(y-x_{0}, \bar{m}(i, j)\right)$. Its value is

$$
\min _{q} \max _{i}\left(y-x_{0}, \sum_{j} q_{j} \bar{m}(i, j)\right)=\min _{q} \max _{t \in T(q)}\left(y-x_{0}, t\right) \geq \min _{s \in S}\left(y-x_{0}, s\right) .
$$

Consequently there is a $p \in P$ such that

$$
\left(y-x_{0}, \sum_{i} p_{i} \bar{m}(i, j)\right) \geqq \min _{s \in S}\left(y-x_{0}, s\right)
$$

for all $j$, that is,

$$
\left(y-x_{0}, r\right) \geqq\left(y-x_{0}, y\right)
$$

for all $r \in R(p)$. Since $\left(y-x_{0}, x_{0}\right)<\left(y-x_{0}, y\right)$, the hyperplane $\left(y-x_{0}, x\right)=$ $\left(y-x_{0}, y\right)$ separates $x_{0}$ from $R(p)$, completing the proof.

On the other hand, any $T\left(q_{0}\right)$ satisfies the hypotheses of Theorem 1 in $M^{\prime}$ with $f: f_{n} \equiv q_{0}$, and so is approachable in $M^{\prime}$ with this $f$. Consequently, if $S$ fails to intersect $T\left(q_{0}\right), S$ is excludable in $M$ with $g: g_{n} \equiv q_{0}$.

COROLlaRY 1. The sets $R(p)$ are approachable with $f: f_{n} \equiv p$.

Corollary 2. A closed convex set $S$ is approachable if and only if for every vector $u$,

$$
v(u) \geq \min _{s \in S}(u, s),
$$

where $v(u)$ is the value of the game with matrix $\|(u, \bar{m}(i, j))\|$.

Proof of Corollary 2. If for some $u_{0}$ the inequality fails, then $T\left(q_{0}\right)$ is disjoint from $S$, where $q_{0}$ is a good strategy for II in the game with matrix $\left\|\left(u_{0}, \bar{m}(i, j)\right)\right\|$, and conversely if any $T\left(q_{0}\right)$ is disjoint from $S$ and $u_{0}$ is a vector with 


$$
\max _{t \in T\left(q_{0}\right)}\left(u_{0}, t\right)<\min _{s \in S}\left(u_{0}, s\right),
$$

then

$$
v\left(u_{0}\right)<\min _{s \in S}\left(u_{0}, s\right)
$$

\section{The case $N=1$.}

THEOREM 4. For $N=1$, let $v, v^{\prime}$ be the values of the games with matrices $M, M^{\prime}$. If $v^{\prime} \leqq v$, a closed set $S$ is approachable if it intersects the closed interval $v^{\prime} v$ and excludable otherwise. If $v^{\prime} \geqq v$, a closed set $S$ is approachable if it contains the closed interval $v v^{\prime}$ and excludable otherwise.

Proof. Application of Corollary 2 to the closed interval $A B, A<B$ with $u= \pm 1$ yields that $A B$ is approachable if and only if $v \geq A$ and $-v^{\prime} \geqq-B$. If $v^{\prime} \leqq v$, these are simply the conditions that $A B$ intersect the closed interval $v^{\prime} v$, and if $v^{\prime} \geqq v$, they are the conditions that $A B$ contain $v v^{\prime}$. Thus if $v^{\prime} \leqq v$ every point in $v^{\prime} v$ is approachable, so that any set $S$ intersecting $v^{\prime} v$ contains an approachable subset and is hence approachable, while if $v^{\prime} \geqq v$, the interval $v v^{\prime}$ and hence any set containing it, is approachable. The last sentence, applied to $M^{\prime}$, yields that if $v^{\prime} \leqq v$, the interval $v^{\prime} v$ is approachable in $M^{\prime}$, so that any closed set not intersecting $v^{\prime} v$ is excludable in $M$, and that if $v^{\prime} \geqq v$, any point in $v v^{\prime}$ is approachable in $M^{\prime}$ so that any closed set not containing $v v^{\prime}$ is disjoint from a point approachable in $M^{\prime}$ and consequently is excludable in $M$. This completes the proof.

5. An example. We saw in the last section that for $N=1$ every set is approachable or excludable. This is false for $N=2$ as is shown by the following example. Let

$$
r=s=2, m(1,1)=m(1,2)=(0,0), m(2,1)=(1,0), m(2,2)=(1,1),
$$

let $I_{1}$ be the set of points $\left(\frac{1}{2}, y\right), 0 \leqq y \leqq \frac{1}{4}$, let $I_{2}$ be the set of points $(1, y), \frac{1}{4} \leqq y \leqq 1$, and let $S=I_{1} \cup I_{2}$. For every $n$, player I has a strategy which guarantees that $\bar{x}_{2 n} \in S$, as follows : $f_{j} \equiv(0,1)$ for $j<n$, so that $\bar{x}_{n}=(u, 1)$; if $u \geq \frac{1}{2}, f_{j} \equiv(0,1)$ for $j \geqq n$, and if $u<\frac{1}{2}, f_{j} \equiv(1,0)$ for $j \geqq n$. Then for $u \geqq \frac{1}{2}, \bar{x}_{2 n} \in I_{2}$, and for $u<\frac{1}{2}, \bar{x}_{2 n} \in I_{1}$. However $S$ is not approachable, since the following strategy for II does permit $\bar{x}_{n}$ to remain near either $I_{1}$ or $I_{2}$. Let $\bar{x}_{n}=\left(a_{n}, b_{n}\right)$, if $a_{n} \geqq \frac{3}{4}, g_{n}=(1,0)$; if $a_{n}<\frac{3}{4}, g_{n}=(0,1)$. Thus $S$ is neither approachable nor excludable.

In the above example, $S$ is weakly approachable, where a set $S$ is said to be weakly approachable in $M$ if for every $\varepsilon>0$ there is an $N_{0}$ such that for every $n \geqq N_{0}$ there is a strategy $f$ for I such that, for all $g$, 
Prob $\left\{\delta_{n}>\varepsilon\right\}<\varepsilon$,

where $\delta_{n}$ is the distance from $\bar{x}_{n}$ to $S$. Similarly $S$ is weakly excludable in $M$ if there is a $d>0$ such that for every $\varepsilon>0$ there is an $N_{0}$ such that for every $n \geqq N_{0}$ there is a strategy $g$ for II such that, for all $f$,

$$
\text { Prob }\left\{\delta_{n}<d\right\}<\varepsilon .
$$

Clearly no $S$ is both weakly approachable and weakly excludable, we conjecture that every $S$ is one or the other. In the above example, it is not hard to show that a closed $S$ is weakly approachable if it intersects the graph of every function $h$ defined for $0 \leqq t \leqq 1$ which satisfies

$$
h(0)=0, \quad 0 \leqq\left(h\left(t_{2}\right)-h\left(t_{1}\right)\right) /\left(t_{2}-t_{1}\right) \leqq 1 \quad \text { for } 0 \leqq t_{1}<t_{2} \leqq 1,
$$

and is weakly excludable if there is such an $h$ whose graph it fails to intersect.

\section{REFERENCES}

1. David Blackwell, On optimal systems, Ann. Math. Stat., 25 (1954), 394-397.

2. John von Neumann and Osker Morgenstern, Theory of games and economic behavior, Princeton, 1944.

HOWARD UNIVERSITY 


\section{PACIFIC JOURNAL OF MATHEMATICS}

\section{EDITORS}

H. L. Royden

Stanford University

Stanford, California

E. HewitT

University of Washington

Seattle 5 , Washington
R. P. Dilworth

California Institute of Technology Pasadena 4, California

E. G. Straus

University of California

Los Angeles 24, California

\section{ASSOCIATE EDITORS}

E. F. BECKENBACH

C. E. BURGESS

H. BUSEMANN

H. FEDERER

\author{
M. HALL \\ P. R. HALMOS \\ V. GANAPATHY IYER \\ R. D. JAMES
}

M. S. KNEBELMAN

I. NIVEN

T. G. OSTROM

M. M. SCHIFFER
J. J. STOKER

G. SZEKERES

F. WOLF

K. YOSIDA

\section{SUPPORTING INSTITUTIONS}

UNIVERSITY OF BRITISH COLUMBIA

CALIFORNIA INSTITUTE OF TECHNOLOGY

UNIVERSITY OF CALIFORNIA

MONTANA STATE UNIVERSITY

UNIVERSITY OF NEVADA

OREGON STATE COLLEGE

UNIVERSITY OF OREGON

UNIVERSITY OF SOUTHERN CALIFORNIA
STANFORD UNIVERSITY

UNIVERSITY OF UTAH

WASHINGTON STATE COLLEGE

UNIVERSITY OF WASHINGTON

AMERICAN MATHEMATICAL SOCIETY CALIFORNIA RESEARCH CORPORATION HUGHES AIRCRAFT COMPANY 


\section{Pacific Journal of Mathematics}

\section{Vol. 6, No. $1 \quad$ November, 1956}

David Blackwell, An analog of the minimax theorem for vector payoffs..... 1

L. W. Cohen, A non-archimedian measure in the space of real

sequences ..................................... 9

George Bernard Dantzig, Constructive proof of the Min-Max theorem ..... 25

Jim Douglas, On the numerical integration of quasilinear parabolic

differential equations ............................... 35

James Michael Gardner Fell, A note on abstract measure ............. 43

Isidore Isaac Hirschman, Jr., A note on orthogonal systems . . . . . . . . . . 47

Frank Harary, On the number of dissimilar line-subgraphs of a given

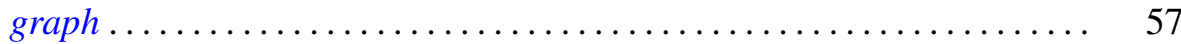

Newton Seymour Hawley, Complex bundles with Abelian group......... 65

Alan Jerome Hoffman, Morris Newman, Ernst Gabor Straus and Olga

Taussky, On the number of absolute points of a correlation ...........

Ernst Gabor Straus and Olga Taussky, Remark on the preceding paper.

Algebraic equations satisfied by roots of natural numbers . . ........ 97

Ralph D. James, Summable trigonometric series ................. 99

Gerald R. Mac Lane, Limits of rational functions . . . . . . . . . . . . . . . 111

F. Oberhettinger, Note on the Lerch zeta function ................. 117

Gerald C. Preston, On locally compact totally disconnected Abelian groups and their character groups ........................... 121

Vikramaditya Singh and W. J. Thron, On the number of singular points, located on the unit circle, of certain functions represented by

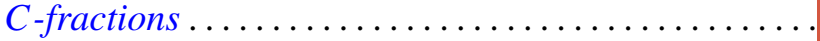

Sherman K. Stein, The symmetry function in a convex body ... 145 Edwin Weiss, Boundedness in topological rings.............

Albert Leon Whiteman, A sum connected with the series for the partition

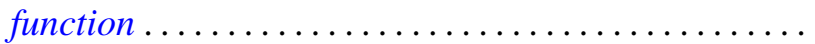

Alfred B. Willcox, Some structure theorems for a class of Banach algebras.

Joseph Lawrence Zemmer, Some remarks on p-rings and their Boolean geometry... 